\title{
Avaliação da tabuada para aprendizagem de adição ou subtração de números Inteiros
}

\section{Table evaluation for learning addition or subtraction of Integers}

Evaluación de tablas para aprender la suma o resta de números enteros

Ramadane Hirage Licenciado em Ensino de Matemática na Universidade Rovuma, Moçambique E-mail ramadane.hirage.3@gmail.com Orcid ID: 0000-0003-4913-2234

Rosalino Subtil Chicote Doutorando em Ensino de Ciências e Educação Matemática - PCI-UEL/UNILICUNGO

E-mail rschicote1@gmail.com Orcid ID: 0000-0003-3454-7816

Geraldo Vernijo Deixa

Doutor em Ensino de Ciências e Educação Matemática

Professor Associado da Universidade Licungo Diretor do Gabinete de Avaliação e Qualidade da Universidade Licungo Coordenador do Mestrado em Educação/Currículo Universidade Licungo, Zambézia, Moçambique E-mail gdeixa@gmail.com Orcid ID: 0000-0002-3992-0993 
Resumo: O objetivo da pesquisa foi apreciar o efeito da incorporação da tabuada de duas operações na aprendizagem de adição ou subtração de Números Inteiros. Selecionou-se aleatoriamente 276 alunos da $8^{a}$ classe da Escola Secundária de Montepuez e esses foram divididos ( $n=138$ ) em dois grupos (experimental e controlo). Aplicou-se o pré-teste aos dois grupos de alunos para verificar o nível de conhecimento. Depois, lecionou-se 3 aulas para cada um dos grupos sendo para o grupo experimental com incorporação da tabuada. Em seguida, aplicou-se o pós-teste aos dois grupos. Através do teste de Mann-Whitney, as medianas do pós-teste foram diferentes. Neste sentido, a mediana do grupo experimental foi igual a 20 e a do grupo de controlo igual a 10. Feitas análises, notou-se que que os alunos que utilizaram a tabuada tiveram mediana superior em relação aos que não se beneficiaram de aulas com tabuada. Conclui-se que a tabuada pode ser usada como meio didático para aprendizagem de Adição ou Subtração de Números Inteiros; seu uso produz efeito positivo na aprendizagem dos alunos.

Palavras-chave: Aprendizagem. Recurso didática. Adição ou Subtração de Números Inteiros na Tabuada

Abstract: The objective of the research was to assess the effect of incorporating the multiplication table of two operations on learning the addition or subtraction of Integers. 276 8th grade students from the Montepuez Secondary School were randomly selected and these were divided ( $n=138$ ) into two groups (experimental and control). The pre-test was applied to the two groups of students to verify the level of knowledge. Afterwards, 3 classes were taught for each one of the groups, being for the experimental group with incorporation of the multiplication table. Then, the post-test was applied to both groups. Using the Mann-Whitney test, the post-test medians were different. In this sense, the median of the experimental group was equal to 20 and that of the control group was equal to 10. After analysis, it was noted that students who used the multiplication table had a higher median compared to those who did not benefit from classes with multiplication tables . It is concluded that the multiplication table can be used as a didactic means for learning Addition or Subtraction of Integers; its use has a positive effect on student learning.

Keywords: Learning. Didactic resource. Addition or Subtraction of Integer Numbers in Times Table

Resumen: El objetivo de la investigación fue evaluar el efecto de incorporar la tabla de multiplicar de dos operaciones en el aprendizaje de la suma o resta de números enteros. Se seleccionaron aleatoriamente 276 estudiantes de octavo grado de la Escuela Secundaria Montepuez y se dividieron $(n=138)$ en dos grupos (experimental y control). La prueba previa se aplicó a los dos grupos de estudiantes para verificar el nivel de conocimientos. Posteriormente se impartieron 3 clases para cada uno de los grupos, siendo para el grupo experimental con incorporación de la tabla de multiplicar. Luego, se aplicó la posprueba a ambos grupos. Usando la prueba de Mann-Whitney, las medianas posteriores a la prueba fueron diferentes. En este sentido, la mediana del grupo experimental fue igual a 20 y la del grupo control fue igual a 10. Luego del análisis, se notó que los estudiantes que usaron la tabla de multiplicar tenían una mediana mayor en comparación con los que no se beneficiaron de clases con tablas de multiplicar. Se concluye que la tabla de multiplicar se puede utilizar como un medio didáctico para aprender Suma o Resta de Enteros; su uso tiene un efecto positivo en el aprendizaje de los estudiantes.

Palabras clave: Aprendizaje. Recurso didáctico. Suma o resta de números enteros en la tabla de multiplicar

Recebido em

23/07/2021.

Aceito em

18/10/2021. 


\section{INTRODUÇÃO}

Ao longo do percurso escolar no Ensino Secundário Geral constatou-se dificuldades enfrentadas pelos alunos (i.e colegas da mesma turma e das outras turmas) em relação à adição e subtração de Números Inteiros na $8^{a}$ classe, onde este conteúdo é introduzido. Sobretudo quando se tratava de números inteiros negativos, com expressões do tipo: "(-2) -(-3)", "(-3) -(+2)”, "(+2)-(+3)".

O Programa do Ensino Geral, INDE (2010), da $8^{\text {a }}$ classe sugere que o professor recorra as situações do dia-a-dia para introduzir e explicar o significado das regras operatórias. Sugere-se com ênfase a abordagem da operação de adição dando o significado quotidiano, o que não acontece com a operação da subtração. Por exemplo, qual é o significado da expressão "(-2) - (-3)" dado que "+" é ganho e "-" é perda e qual a diferença na explicação com " $(-2)+(+3)$ ".

A mesma situação verifica-se nos livros didáticos. Nos livros dá-se ênfase aos jogos de sinais. Essas sugestões justificam o questionamento seguinte: como se explica que uma perda multiplicada com uma perda se transforma num ganho. (Deixa \& Passos, 2013).

Enfim, a maior parte dos alunos revelam dificuldades em operar com a subtração de números inteiros comparativamente à adição. Cid (2003) falando de competências de alunos para a realização de operações formais, faz referência de algumas pesquisas que há evidências de melhores resultados nas questões envolvendo adição, e a operação de subtração de inteiros com questões do tipo: "(-5) - (+3)" ou "(4) - (-3)" apresentam o menor índice de acertos.

Esses obstáculos se agregam a outros provocando baixo desempenho e impedindo o aluno de progredir. Para Gonçalves (2007), se as dificuldades na aprendizagem de Números Inteiros não forem superadas nas classes introdutórias arrastam-se pelos anos seguintes, provocando lacunas no aprendizado. Deixa (2014) argumenta que para o caso de Moçambique existem lacunas no ensino e 


\section{Universidade Federal da Grande Dourados}

aprendizagem de números inteiros. O mesmo autor enfatiza a necessidade de pesquisar acerca do assunto.

Fora do contexto moçambicano, algumas pesquisas procuram trazer soluções para as dificuldades. De modo geral, elas convergem em cinco grupos: o uso de jogo (Ferreira, 2018); o uso da reta numérica (Martini, 2010); material manipulativo como quadradinhos (Bordin, 2011); o uso de tecnologia como calculadora (Salgado,2011); e a etnomatematica (Soares, 2007). Apesar de crescentes propostas, não deixam de sugerir a criatividade e necessidade de buscar inovações no que tange recursos didáticos para aprendizagem de Números Inteiros Negativos. Um estudo realizado por Correia (2017) evidencia isso.

[...] percebe-se a necessidade de buscar inovações para o ensino não somente de números inteiros, mas da Matemática no geral, adotando novos métodos e ferramentas educacionais que estimulem $o$ interesse e a curiosidade dos estudantes, o que é fundamental para que o aluno seja capaz de construir seu próprio conhecimento. [...]. (p.89)

As pesquisas e situações descritas destacam que a aprendizagem dos números inteiros é insatisfatória. Levando em consideração a recomendação de Correia (2017) desenvolveu-se uma tabuada para ensino da adição ou subtração de números inteiros.

A pesquisa procurou apreciar o efeito da incorporação da tabuada de duas operações na aprendizagem de adição ou subtração de Números Inteiros para alunos da $8^{\text {a }}$ classe. A variável independente foi o tipo de aula (com ou sem uso da tabuada de duas operações). A variável dependente foi a aprendizagem dos alunos denotada através da comparação entre as medianas das notas.

A pesquisa teve como hipóteses:

Hipótese nula: Não há diferença estatisticamente significativa entre a mediana das notas do grupo de alunos que tiveram aulas de adição ou subtração de números relativos com uso da tabuada de duas operações e o grupo que tiveram aulas sem uso da tabuada. 
Hipótese alternativa: Há diferença estatisticamente significativa entre a mediana das notas do grupo de alunos que tiveram aulas de adição ou subtração de números relativos com uso da tabuada de duas operações e o grupo que tiveram aulas sem uso da tabuada.

\section{FUNDAMENTAÇÃO TEÓRICA}

Nesta secção inicia-se por situar a perspectiva de aprendizagem adotada na pesquisa. Em seguida, procuramos desvelar que a tábua da adição ou subtração se enquadra na classe de recursos didáticos.

\subsection{SOBRE A APRENDIZAGEM}

A aprendizagem, segundo Tavares e Alarcão (2005, p. 86), é " uma construção pessoal, resultante de um processo experimental, interior à pessoa e que se traduz numa modificação de comportamento relativamente estável". Assim, a aprendizagem é experiencial, interior à pessoa, na medida em que apenas pode-se avaliar a aprendizagem através das modificações exteriores que ela apresenta. É através das manifestações exteriores que se vê se o sujeito aprendeu, mas estas só se revelam se no interior do sujeito tiver havido um processo de mudança relativamente permanente. (Tavares \& Alarcão, 2002).

Segundo Pinto (2003), a aprendizagem é um processo de transferência de uma situação inicial (a situação atual em termos de competências, saberes sobre adição e subtração de números inteiros negativos usando outros meios, por exemplo, para o estado final (novas competências ou saberes da temática) através de experiência (incorporação da tabuada na adição e subtração de números negativos, por exemplo).

Segundo os behavioristas, aprendizagem ocorre através dos relacionamentos de dois eventos, estímulo e respostas, ou seja, de respostas que individuo dá depois receber estímulos externos (Moreira, 2016). Neste caso, a lecionação de aulas sobre 


\section{Universidade Federal da Grande Dourados}

adição e subtração com incorporação da tabuada seria um dos estímulos externo e, como resposta será o desempenho em termo de notas a partir do teste ou um diagnóstico.

A proposta do Skinner vai além dos dois eventos. Ele introduz as variáveis de entrada (estímulo, reforço, e contingências de reforço) e saída (respostas). Na concepção de Skinner, segundo Moreira (2016), as variáveis de saída são as respostas do sujeito, as quais podem ser respondentes ou operantes. As operantes são aquelas nas quais o sujeito faz algo que tem um efeito no mundo exterior, que opera nele. A cada tipo de resposta, respondente ou operante, corresponde a um tipo de condicionamento (um procedimento) que, em segundo caso, do introduzir um reforçador positivo imediatamente após uma resposta, resultando em um aumento de frequência dessa resposta.

Na perspectiva de Skinner, o condicionamento operante é um ato de introduzir um reforçador depois de uma resposta desejada, provocando em aumento de frequência dessa resposta.

Os recursos didáticos, as aulas, os materiais programados funcionam como estímulos para as respostas desejadas, [...]. O professor atua tecnicamente, procurando modificar ou implantar comportamentos através de condicionamentos, procedimentos de extinção, modelagens, reforçamentos diferenciais, ainda que implicitamente ou inconscientemente (Moreira, 2016, p.10-11).

Nesta vertente, se professores de matemática introduzir um reforçar (a tabuada de duas operações, por exemplo), na aula de adição ou subtração de números Inteiros Negativos poderia provocar repetição de respostas desejadas facilitando assim aprendizagem dos alunos.

\subsection{RECURSO didÁtICO e ApRENDIZAGEM DE MATEMÁtICA}

Segundo Cerqueira e Ferreira (2007) recursos didáticos

"[...] são todos os recursos físicos, utilizados com maior ou menor frequência em todas as disciplinas, áreas de estudo ou atividades, sejam quais forem as 
técnicas ou métodos empregados, visando auxiliar o educando a realizar sua aprendizagem mais eficientemente, constituindo-se num meio para facilitar, incentivar ou possibilitar o processo ensino-aprendizagem." ( $p, 1)$.

Os termos como recurso didático, material didático, recurso educativo, material manipulável, material concreto e meio didático "surgem com sentidos sobrepostos, apesar de não terem exatamente o mesmo significado. Com efeito, as várias definições encontradas para o conceito não divergem muito umas das outras, ocorrendo com alguma sobreposição de significados" (Botas \& Moreira, 2013, p.255). Eles "são usados pelos professores para descrever os instrumentos utilizados como facilitadores da aprendizagem" (Passos \& Takahashi, 2018, p.175).

Percebe-se que os recursos didáticos são todos os meios ou materiais, como jogos pedagógicos, tabuada, ou outros, utilizados na disciplina de matemática ou, em qualquer outra área de estudo, com objetivo de facilitar, incentivar, auxiliar eficientemente o ensino e aprendizagem da matemática e em particular da adição ou subtração de Números Inteiros por exemplo, e como alternativa metodológica á disposição do professor e do educando.

Nas aulas de Matemática, os recursos didáticos "[...] devem servir como mediadores para facilitar a relação professor/aluno/conhecimento no momento em que um saber está sendo construído” (Passos, 2004, p. 1). “ [...]. Aliás, esse deve ser o objetivo maior de sua utilização nos processos de ensinar e de aprender Matemática" (Nogueira, Ferreira \& Oliveira 2010, p.7), portanto, os recursos didáticos devem servir para provocar debates em sala de aula.

\section{PROCEDIMENTOS METODOLÓGICOS}

Escolheu-se pesquisa quantitativa como perspetiva de abordagem do problema. A escolha deste tipo de pesquisa deveu-se o facto de comparação de medianas das notas do grupo de experimento e de controlo. Na comparação das medianas das notas dos dois grupos requer o uso de testes estatísticos (Prodanov \& Freitas, 2013).

Conforme o Programa de Ensino de Matemática da $8^{a}$ classe, os conteúdos são divididos em três trimestres (a carga horária total dos três trimestres é 120), sendo 
que no primeiro trimestre são tratados conteúdos de Números Racionais (inclui estudo de Números Inteiros) e Equações Lineares; no segundo, Proporcionalidade e funções lineares, e Sistema de duas equações lineares a duas incógnitas; no terceiro e último, Circunferências e Círculos, Congruência de triângulos e Teorema de Pitágoras. Neste caso, os Números Inteiros são os primeiros conteúdos que os alunos estudam após entrada na $8^{a}$ classe, no $1^{\circ}$ ciclo do Ensino Secundário. O $1^{\circ}$ ciclo é constituído por três classes, a saber, 8ª $9^{\underline{a}}$ e $10^{\underline{a}}$ classe.

A pesquisa foi realizada com 276 alunos da $8^{\text {a }}$ classe de ambos sexos (com 13 à 15 anos de idades) da Escola Secundária de Montepuez, no primeiro trimestre de 2020. Esses alunos foram escolhidos aleatoriamente. Para tal, atribui-se um número a cada aluno e selecionou-se através da tabela de números aleatórios os alunos da amostra pretendida. Utilizou-se os números aleatórios da $1^{\underline{a}}$ à $24^{\underline{a}}$ coluna e da $1^{\underline{a}}$ à $40^{\underline{a}}$ linha. Porém, das colunas 21 à 24 usou-se apenas $1^{\underline{a}}$ linha até a $7^{\text {ạ }}$ linha, pois os números precisados já estavam completos.

Para a formação de grupo de controlo e grupo experimental foi utilizado um sorteio de papeis que foram baralhados num recipiente. Esses papeis foram de um número igual ao tamanho da amostra (de 276 alunos) determinado e selecionado anteriormente e cada papel possuía o número aleatório que identificava o aluno.

Após os papeis serem baralhados num recipiente, foram retirados um por um e colocados em duas caixas. Uma caixa escrita grupo experimental (G.E) e a outra escrita grupo de controlo (G.C). A cada caixa era colocada uma metade dos papeis. Sendo assim, em cada processo de seleção dos grupos de estudo não precisou a presença dos alunos. Com esse processo, 276 alunos foram divididos em duas partes iguais de modo que a primeira parte constituir alunos do grupo de controlo (138) e outra a segunda parte, alunos do grupo experimental (138).

Depois de se formar o grupo de controlo e experimental, marcou-se dias, horas e sala onde houve lecionação de aulas por pesquisadores e realização dos testes escritos. Foram 9 dias, dentre esses, 6 dias foram de lecionação de aulas. 
Primeiro dia realizou-se o pré-teste para os dois grupos (i.e. grupo experimental e de controlo) a fim de identificar o nível de conhecimento que os alunos se encontravam em relação ao conteúdo de Adição e Subtração em Números Inteiros. Neste teste, os alunos deviam aplicar os conhecimentos que tiveram com os seus professores e os possíveis procedimentos didáticos aprendidos.

No segundo, terceiro e quarto dia, lecionou-se uma aula para o grupo de controlo sobre Adição e Subtração em Números Inteiros aplicando os meios presentes nos manuais didáticos com objetivo de fazer uma breve revisão do conteúdo visto pelos alunos. Importa clarificar que os alunos já tiveram esse conteúdo com seus professores anteriormente, antes da realização do pré-teste. No fim da aula realizouse alguns exercícios e tarefas para os alunos irem resolver em casa. No quinto dia, a resolução de trabalho de casa e em seguida um pós-teste para o mesmo grupo de controlo.

No sexto, sétimo e oitavo dia realizou-se duas novas aulas para o grupo experimental tendo os seguintes objetivos: construir a tabuada, efetuar as operações de Adição e Subtração e Adição algébrica de números Inteiros usando a tabuada. Em cada momento os alunos aprenderam a construir a tabuada, realizaram operação usando a tabuada, como forma de exercitação, tiveram exercícios para resolver na sala de aula junto com os colegas e tarefas como trabalho para casa de modo a se preparar para o pós-teste.

Por último, no nono dia, realizou-se um pós-teste para o grupo de experimento. $\mathrm{O}$ teste foi o mesmo tanto para o grupo de controlo assim como, para o experimental. $E$ cada aula tinha a duração de 45 minutos. A partir do que se realizou nas secções anteriores concluiu-se que a pesquisa toma o carácter experimental.

Um pré-teste aplicado a todos os grupos, que tinha como objetivo diagnosticar o nível de conhecimento dos alunos. E o pós-teste visando comparar os resultados do teste nos dois grupos de alunos de modo a apreciar a aprendizagem de adição e subtração em relação às duas metodologias usados pelos alunos. 


\section{Universidade Federal da Grande Dourados}

Esses testes tinham 4 questões diferentes, tendo como cotação 5 valores a cada. Uma questão referente adição, uma subtração e duas referentes a soma algébrica. Porém, no pós-teste o cenário foi pouco diferente devido aos resultados obtidos na tarefa referente adição, por isso, foram duas questões referente a subtração e duas somas algébrica.

Importa afirmar que as tarefas do pré-teste foram diferentes das do pós-teste nos dois grupos de modo a garantir que os resultados dos alunos no pré-teste não influenciassem no pós-teste.

$\mathrm{Na}$ correção das questões dos testes, constatou-se que havia três grupos de alunos em relação aos resultados obtidos: os que deixaram em branco, erraram e acertaram. Os alunos que deixaram em branco considerou-se valor zero (0). Os que erraram são aqueles que tentaram resolver, mas não corretamente, os seus valores variam de um (1) à quatro (4) conforme o esforço. E os que acertaram resolveram corretamente e tiveram cinco (5) valores.

As questões propostas para o pré-teste foram:
$Q_{1}:(+2)+(+6)=$
$Q_{2}:(-5)-(-2)=$
$Q_{3}:(+1)-(+8)=$
$Q_{4}:(+5)-(-3)+(-9)=$

A questão 1, o objetivo era de diagnosticar se os alunos tinham o domínio e perceção de adição de Números Inteiros. E a tarefa dos alunos consistia em adicionar os dois números.

Para as questões 2 e 3 , o objetivo destas era de diagnosticar o conhecimento de subtração de Números Inteiros aos alunos. Os alunos deviam subtrair os dois números conforme aprenderam com os seus professores.

A questão 4, o objetivo era de diagnosticar se os alunos sabiam efetuar adição algébrica, sendo assim, a tarefa do aluno era de conjugar os sinais e adicionar ou subtrair os números compostos na expressão conforme aprendido. 
O pós-teste foi realizado depois de aulas lecionadas pelos pesquisadores para os dois grupos. O teste tinha quatro (4) questões.
$Q_{1}:(+5)-(+3)=$
$Q_{2}:(-8)-(-6)=$
$Q_{3}:(+3)-(+1)+(-7)=$
$Q_{4}:(-4)-(-2)+(-5)-(-3)=$

Nas questões 1 e 2, tinha o propósito de avaliar se os alunos com ou sem aulas experimentais de tabuada conseguem subtrair os dois números inteiros. A atividade dos alunos era de subtraírem consoante aprenderam nas aulas lecionadas pelos pesquisadores.

Diferentemente das questões anteriores, as questões 3 e 4 era de aferir se alunos com ou sem o uso da tabuada conseguem efetuar adição algébricas de números inteiros. O trabalho do aluno era de conjugar os sinais e adicionar ou subtrair os números contidos na expressão conforme aprendido.

Calculou-se o coeficiente alfa de Cronbach com ajuda de aplicativo estatístico SPSS versão 22, a fim de avaliar o grau de consistência, de estabilidade e a precisão do instrumento. Sendo assim, os valores encontrados foram 0,731 no pré-teste e 0,795 no pós-teste. E esses valores estão no intervalo satisfatório.

Com o mesmo aplicativo Estatístico SPSS, testou-se a normalidade de distribuição das variáveis com ajuda do teste de Kolmogorov-Sminov. E para homogeneidade das variâncias recorreu-se o teste de Levene. Assim com um nível de significância de 5\%, os $p$-value obtidos para pré-teste e pós-teste foram de 0,00 , que significa que as variáveis não são normalmente distribuídas nem as variâncias são homogéneas nas duas amostras.

Por causa dos resultados encontrados do teste de normalidade e da homogeneidade das variâncias, permitiu-se optar teste Mann-Whitney para testar as hipótese nula. Essa hipótese foi validada, com um nível de confiança de 95\%, p-value encontrado foi superior a $5 \%$.

O teste de Mann-Whitney utilizou-se no pré-teste na comparação das medianas dos dois grupos com objetivo de identificar o nível de conhecimento dos alunos em 
relação a adição e subtração de Números Inteiros sem uso da tabuada. E na comparação das medianas das notas do pós-teste a fim de apreciar aprendizagem da temática com incorporação da tabuada.

\subsection{Procedimento para construção da tabuada de duas OPERAÇÕES}

10 passo: Constrói-se uma tabela, onde na parte vertical são colunas e a parte horizontal são linhas. O número de colunas pode ser ou não iguais ao número de linhas, dependerá dos números que se quer operar (adicionar ou subtrair). No quadradinho da $1^{\underline{a}}$ linha e $1^{\underline{a}}$ coluna coloca-se sinal de operação.

20 passo: Inicia-se por preencher a tabela com os zeros na diagonal principal.

3o passo: Partindo da noção de Números Inteiros, onde os números acima e direita do zero são positivos e os que estão abaixo e a esquerda do zero são números negativos. Com os mesmos procedimentos, preenche-se todos os

\begin{tabular}{|c|c|c|c|}
\hline$+/-$ & 1 & 2 & 3 \\
\hline-1 & 0 & 1 & 2 \\
\hline-2 & -1 & 0 & 1 \\
\hline-3 & -2 & -1 & 0 \\
\hline
\end{tabular}
quadradinhos da tabela.

Como fazer operação: primeiro, deve-se localizar o quadradinho da primeira parcela (primeiro número) e em seguida, conta-se tantos quadradinhos do valor da outra segunda parcela (segundo número), onde termina a contagem, obtém-se assim o resultado da operação.

Caso tiver mais de duas parcelas na expressão, conta-se tantos quadradinhos a partir de onde termina a segunda parcela (segundo número).

Para isso, deve-se seguir as seguintes regras:

1ª Regra: Se o número for positivo, deve-se contar os quadradinhos para cima ou para direita. 
2a Regra: Se o número for negativo, deve-se contar os quadradinhos para baixo ou para esquerda.

Nota importante: 1를 Se o número for negativo precedido com um sinal operacional negativo segue-se a primeira regra e $2^{\mathrm{a}}$ : Se número for positivo precedido com um sinal operacional negativo segue-se a segunda regra.

Exemplo: $(+2)-(+3)=-1$

Para este caso, tem-se muitas possibilidades. Uma delas, escolhe-se 2 (primeira parcela) da primeira linha terceira coluna, conta-se quadradinhos (segunda parcela) para baixo. Assim obtém-se o resultado -1 .

Portanto, o uso da tabuada permite obter

\begin{tabular}{|c|c|c|c|}
\hline+ & 1 & 2 & 3 \\
\hline-1 & 0 & 1 & 2 \\
\hline-2 & -1 & 0 & 1 \\
\hline-3 & -2 & $-1^{\downarrow}$ & 0 \\
\hline
\end{tabular}
algumas características que ajudam a inferir algumas regras para adição e subtração números inteiros:

\subsubsection{Regras de adição}

- A adição de dois números inteiros com o mesmo sinal posicional é outro número inteiro com o mesmo sinal e cujo valor absoluto é igual à soma dos valores absolutos das parcelas.

Exemplo: $(+2)+(+3)=+5$

$(-2)+(-3)=-5$

- A adição de dois números inteiros com sinais posicionais diferentes é também um número inteiro, com valor absoluto igual à diferença dos valores das parcelas $\mathrm{e}$ sinal igual ao da parcela com maior valor absoluto.

$$
\text { Exemplo: }(-2)+(+3)=+1 \quad(+2)+(-3)=-1
$$

\subsubsection{Regras de Subtração}

- A subtração de dois números inteiros com o mesmo sinal posicional é outro número inteiro, com valor absoluto igual à diferença dos valores das parcelas e sinal contrário ao das parcelas. 


\section{Universidade Federal da Grande Dourados}

Exemplo: $(-2)-(-3)=+1 \quad(+2)-(+3)=-1$

- A subtração de dois números inteiros, com sinais posicionais diferentes é também um número inteiro, cujo o valor absoluto é igual à soma dos valores das parcelas e sinal igual ao da primeira parcela.

Exemplo: $(+2)-(-3)=+5 \quad(-2)-(+3)=-5$

\section{RESULTADOS E DISCUSSÃO}

Nessa secção, apresentam-se os resultados do pré-teste e do pós-teste. As apresentações são feitas simultaneamente com as discussões.

\subsection{O Resultado do PrÉ-Teste}

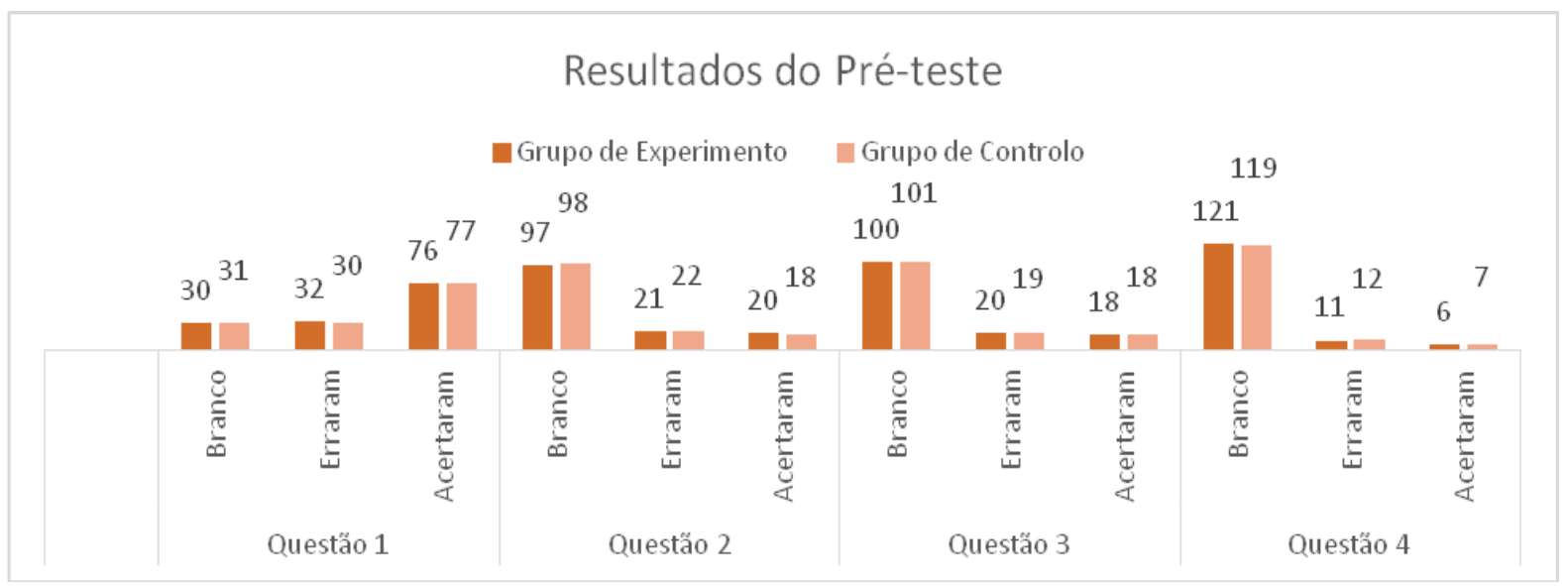

Figura 1: Os resultados obtidos no pré-teste

Fonte: dados da pesquisa, 2021.

Na questão 1, do gráfico a cima, dos 276 alunos que realizaram o diagnóstico, 153 resolveram corretamente, 61 deixaram em branco e 62 erraram. Maior número dos alunos acertou corretamente em relação aos que erraram.

Cid (2003) e Soares (2008), nas suas pesquisas constataram os mesmos resultados nas tarefas relacionadas à adição. Neste caso, a adição de números 


\section{Universidade Federal da Grande Dourados}

inteiros ainda permanece como sendo a operação que os alunos apresentam melhores resultados.

Para as questões 2, 3 e 4, conforme ilustra o gráfico a cima, o maior número dos alunos pertencentes nos dois grupos deixou em branco. E foram minoria dos alunos que resolveram corretamente.

Os erros na subtração de Números Inteiros não são algo recentes. Silveira (2017), Martínez (2018) nos seus estudos sobre aprendizagem dos números inteiros e erros que os alunos apresentam na resolução das atividades, os maiores erros persistentes nas práticas operacionais foram da subtração. Banzatto (2002) fez estudo com uma das tarefas do gênero. Com muitos erros observados, verificou que a subtração é complexa, quando o aditivo é menor que o subtrativo.

Segundo Caraça (1963 apud Hillesheim \& Moretti, 2013) no campo relativo, a soma e/ou subtração de duas operações que aparecem assim unificadas numa só, se chama adição algébrica. De acordo com Soares (2008), ao trabalhar com a soma algébrica de números inteiros negativos foi possível verificar o baixo desempenho. mesmo cenário foi constatado por Gonçalves (2007).

De forma geral, se considerar a quantidade dos alunos que tiveram menor desempenho na subtração e na adição algébrica em relação a dos alunos que tiveram resultados positivos, pode-se afirmar que os alunos apresentam lacunas nas questões relacionadas à subtração e na adição algébrica de Números Inteiros.

Em seguida, comparou-se as medianas das notas do pré-teste dos dois grupos, partindo-se das hipóteses, com um nível de significância de 0,05.

Hipótese nula: Não há diferença estatisticamente significativa entre a mediana do grupo de controlo e a do grupo experimental.

Hipótese alternativa: Há diferença estatisticamente significativa entre a mediana do grupo de controlo e a do grupo experimental. 
Resumo de Teste de Hipótese

\begin{tabular}{|c|c|c|c|}
\hline Hipótese nula & Teste & Sig. & Decição \\
\hline $\begin{array}{c}\text { A distribuição de notas do pré- } \\
\text { teste é a mesma entre as } \\
\text { categorias de grupo de estudo. }\end{array}$ & $\begin{array}{c}\text { Teste U de Mann- } \\
\text { Whitney de amostras } \\
\text { independentes }\end{array}$ &, 056 & $\begin{array}{c}\text { Reter a } \\
\text { hipótese nula }\end{array}$ \\
\hline
\end{tabular}

Sâo exibidas sgnificâncias assintóticas. 0 nível de significância é ,05.

Figura 2: Teste de Mann-Whitney sobre os resultados do pré-teste

Fonte: Organizado pelos autores, 2021.

A decisão (Reter a hipótese nula) do teste de Mann-Whitney significa que a hipótese acima foi aceite, que isso, mostra que não há diferença estatisticamente significativa entre a mediana do grupo de controlo e a do grupo experimental, conforme ilustra a figura 2. Isto é, a concentração das notas é a mesma entre os dois grupos de estudo. Portanto, os alunos dos dois grupos de estudos se encontravam na mesma situação em termo de aprendizagem.

\subsection{Os Resultados do Pós-Teste}

O pós-teste foi realizado depois de aulas lecionadas pelos pesquisadores para os dois grupos. O teste tinha propósito de a apreciar se os alunos com ou sem aulas experimentais de tabuada conseguem subtrair os números inteiros ou realizar adição algébrica. O gráfico abaixo apresenta a comparação descritiva das medianas entre os dois grupos: (controle - alunos que tiveram aulas sem uso da tabuada; experimental - alunos que tiveram aulas com uso da tabuada de duas operações). 


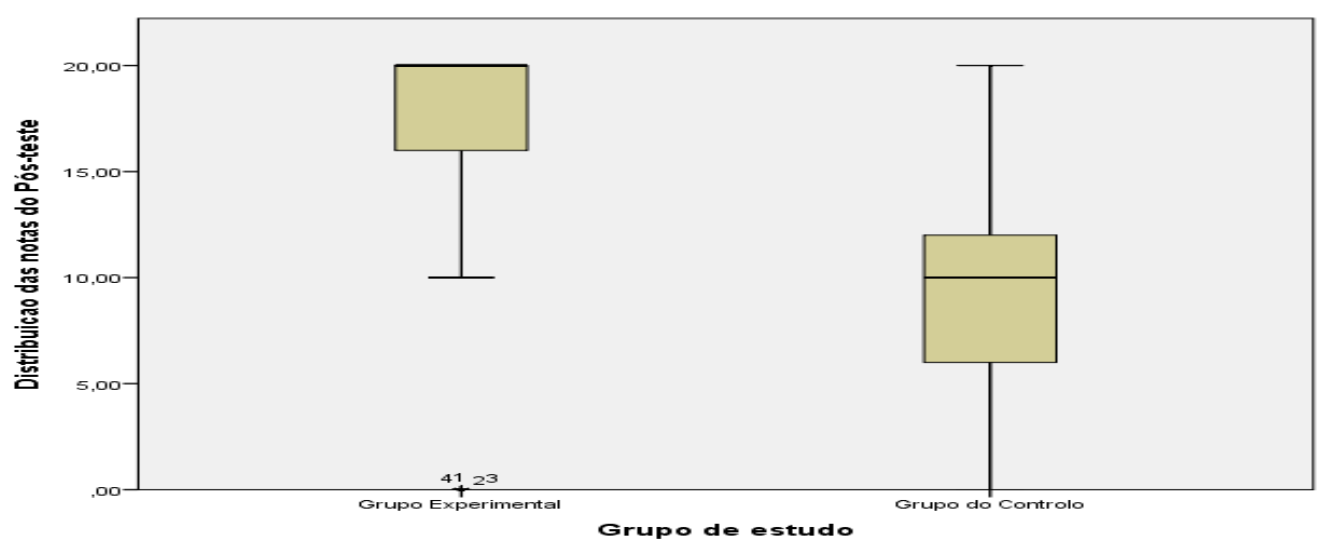

Figura 3: Resultados das medianas das questões de Pós-teste Fonte: dados da pesquisa, 2021.

Com base na leitura da figura 3 a cima, verifica-se que no grupo experimental, no teste, a mediana foi superior em relação ao grupo de controlo. A mediana do grupo experimental coincide no $3^{\mathrm{a}}$ quartil do grupo de controlo, e que $50 \%$ das notas dos alunos experimentais foi de 20 valores e as restantes $50 \%$ variaram no intervalo de 15 a 20 valores. Para o grupo de controlo aconteceu de forma inversa, $50 \%$ das notas dos alunos se concentraram abaixo de 10 valores e 50\% variam a baixa de 15 e a cima de 10. Sendo assim, os alunos pertencentes ao grupo experimental tiveram apresentaram notas elevadas em relação ao outro grupo de alunos.

Soares (2007), Martini (2010), Bordin (2011), Salgado (2011), Silveira (2017) e Ferreira (2018) depois de constatarem algumas dificuldades dos alunos sobre operação de adição e subtração de Números Inteiros, aplicaram propostas didáticas, uso de jogos e materiais didáticos. Com aplicação foi possível realizar reflexões de alguns aspetos influentes no desempenho da aprendizagem dos alunos. Também, verificaram que inovar durante as aulas, trazendo propostas diferentes e mais dinâmicas no ensino de números inteiros, com atividades em grupo e individual, e com materiais atrativos, provocam um impacto positivo na aprendizagem dos alunos. Nas pesquisas precedentes, os alunos que utilizaram as propostas tiveram acertaram mais. 


\section{Universidade Federal da Grande Dourados}

Para ter acurácia do resultado acima exposto por essa pesquisa, consideraramse as seguintes hipóteses:

Hipótese nula: Não há diferença estatisticamente significativa entre a mediana das notas do grupo de alunos que tiveram aulas de adição e subtração de números relativos com uso da tabuada de duas operações e o grupo que tiveram aulas sem uso da tabuada.

Hipótese alternativa: Há diferença estatisticamente significativa entre a mediana das notas do grupo de alunos que tiveram aulas de adição e subtração de números relativos com uso da tabuada de duas operações e o grupo que tiveram aulas sem uso da tabuada.

A análise realizada pelo teste de Mann-Whitney, a um nível de significância de 0,05 sugere a rejeição da hipótese nula, dado que os valores de sig encontrados são inferiores a 0,05 como ilustra o output da figura 4 abaixo.

\section{Resumo de Teste de Hipótese}

\begin{tabular}{|c|c|c|c|c|}
\hline & Hipótese nula & Teste & Sig. & \multicolumn{2}{|l}{ Decisão } \\
\hline $\mathbf{1}$ & $\begin{array}{c}\text { A distribuição de total de notas do } \\
\text { pós-teste é a mesma entre as } \\
\text { categorias de grupo de estudo. }\end{array}$ & $\begin{array}{c}\text { Teste U de Mann- } \\
\text { Whitney de amostras } \\
\text { independentes }\end{array}$ &, 000 & $\begin{array}{l}\text { Rejeitar a } \\
\text { hipótese } \\
\text { nula }\end{array}$ \\
\hline
\end{tabular}

São exibidas significâncias assintóticas. 0 nível de significância é ,05.

Figura 4: Teste de Mann-Whitney sobre os resultados do pós-teste

Fonte: Organizado pelos autores, 2021.

A decisão do teste (rejeitar a hipótese nula) significa que a hipótese nula não foi verificada, neste caso, fica a hipótese alternativa que sustenta que há diferença estatisticamente significativa entre a mediana das notas do grupo de alunos que tiveram aulas de adição e subtração de números relativos com uso da tabuada de duas operações e o grupo que tiveram aulas sem uso da tabuada

Neste caso, uma aprendizagem significativa dos alunos na adição e subtração depois de uma intervenção pedagógica não constituí um cenário novo em outras 
pesquisas. O mesmo se verificou depois da incorporação da tabuada. Sinalizando, assim que as propostas possuem seu potencial formativo.

A tabela a baixo ainda mostra a mudança do desempenho dos alunos dos dois grupos entre o pré-teste e o pós-teste em termo de medianas.

Tabela 1: As medianas do Pré e Pós-teste dos grupos

\begin{tabular}{|c|c|c|}
\hline Grupo de estudo & Total de notas do Pré-teste & Total de notas do Pós-teste \\
\hline Grupo Experimental & 5,0 & 20,0 \\
Grupo do Controlo & 5,0 & 10,0 \\
Total & 5,0 & 16,0 \\
\hline
\end{tabular}

Fonte: organizado pelos autores, 2021.

Segundo Pinto (2003) aprendizagem é a "modificação estável no comportamento do sujeito devido à sua experiência" (p.13) e é através das manifestações exteriores que se vê se o sujeito aprendeu, mas estas só se revelam se no interior do sujeito tiver havido um processo de transformação e mudança. (Tavares \& Alarcão, 2002)

Observando a tabela 1, verifica-se que no grupo experimental, as medianas obtidas no pós-teste superam as do pré-teste. Nesta situação, nota-se uma mudança significativa no grupo. Com base ao conceito de aprendizagem, infere-se que os alunos de grupo experimental aprenderam com facilidade a Adição e Subtração de Números Inteiros; aprendizagem resultante da utilização de tabuada.

\section{CONSIDERAÇÕES FINAIS}

Com base nos resultados, verificou-se que nas 4 (quatro) questões propostas no pré-teste, maior número dos alunos teve dificuldades durante a resolução das questões relacionadas à subtração e adição algébrica, utilizando as metodologias aprendidas e patentes nos livros didáticos.

Com ajuda do teste estatístico de Mann-Whitney, foi possível verificar que esses alunos estavam na mesma situação em termo de nível de aprendizagem no momento do pré-teste, dado que as medianas dos dois grupos foram estatisticamente iguais. 


\section{Universidade Federal da Grande Dourados}

Já no pós-teste, também, com ajuda do Teste de Mann-Whitney, foram testadas hipótese nula: não há diferença estatisticamente significativa entre a mediana das notas do grupo de alunos que tiveram aulas de adição ou subtração de números relativos com uso da tabuada e o grupo que tiveram aulas sem uso da tabuada; hipótese alternativa: há diferença estatisticamente significativa entre a mediana das notas do grupo de alunos que tiveram aulas de adição ou subtração de números relativos com uso da tabuada de duas operações e o grupo que tiveram aulas sem uso da tabuada. O nível de significância adotado foi de 0,05.

Como resultado, o teste estatístico de Mann-Whitney rejeitou a hipótese nula e foi aceite a hipótese alternativa. E tendo em conta que a mediana das notas do aluno que tiveram aulas com uso da tabuada foi superior em relação a do grupo de aulas que tiveram aula sem uso da tabuada, conclui-se que a tabuada pode ser usada como meio didático para aprendizagem de Adição ou Subtração de Números Inteiros; seu uso produz efeito positivo na aprendizagem dos alunos. Nestes termos, sugere-se a constituição de um programa de desenvolvimento profissional de professores de matemática com a finalidade de os habilitar no uso da tabuada.

\section{REFERÊNCIAS BIBLIOGRÁFICAS}

Banzatto, G. B. (2002). Educação Matemática e investigação-ação: aprendendo problemas aditivos com números negativos junto aos meus alunos. UNIMEP. Educação Matemática, n.19

Bordin, L. M. (2011). Os materiais manipuláveis e os jogos pedagógicos como facilitadores do processo de ensino e aprendizagem das operações com números inteiros. (Dissertação de Mestrado em Ensino de Matemática). Universidade Franciscana, Santa Maria. 


\section{Universidade Federal da Grande Dourados}

Botas, D., \& Moreira, D. (2013). A utilização dos materiais didáticos nas aulas de Matemática - Um estudo no $1^{\circ}$ Ciclo. Revista Portuguesa De Educação. V 26(1). pp. 253-286. Recuperado de: https://doi.org/10.21814/rpe.3259

Cerqueira, J. B., \& Ferreira, E. M. B. (2007). Recursos Didácticos na Educação Especial. Instituto Benjamin Constante. Rio de Janeiro.

Cid, E. (2003). La investigación didáctica sobre los números negativos: estado de la cuestión. Pre-publicaciones del seminario matemático "garcia de galdeano". Universidad de Zaragoza. Zaragoza.

Correia, L. P. (2017). Uma intervenção no ensino de operações com números inteiros . (Dissertação de Mestrado em Matemática). Universidade Estadual do Norte Fluminense Darcy Ribeiro. Centro de Ciência e Tecnologia. Laboratório de Ciências Matemáticas. Campos dos Goytacazes.

Deixa, G. V. (2014). Uma abordagem dos números inteiros relativos na $8^{\mathrm{a}}$ classe: indicadores para uma proposta de formação de professores. (Tese para obtenção de grau do titulo de Doutor em Ensino e Educação Matemática). Universidade Estadual de Londrina, Londrina.

Deixa, V. G \& Passos, M. M. (2013). A experiência de 4 professores a respeito do ensino dos números inteiros: um estudo com professores moçambicanos. In Anais do XI Encontro Nacional de Educação Matemática. Curitiba, Paraná. 


\section{Universidade Federal da Grande Dourados}

Ferreira, G. S. (2018). O ensino de adição e subtração de números inteiros a partir de um jogo. (Monografia para obtenção do grau de Licenciatura em Matemática). Universidade Federal de Uberlândia, Uberlândia.

Gonçalves, R. S. (2007). Um Estudo com Números Inteiros usando o Programa Aplusix com alunos de 6ª série do Ensino Fundamental. (Dissertação para obtenção do Mestre Profisional em Ensino de Matematica). Pontificia Universidade Católica de Sao Paulo, São Paulo.

INDE. (2010). Programa da Matemática da 8ํㅡㄹ Classe. Maputo: MEC/INDE.

Martinez, Y. (2018). Errores y dificultades que presentan los alunos de 2 do ano de secundaria en la resolución de atividades com números enterros. (Tesina para la obtencion de la Licenciatura en Ensenanza de la Matematica). Universidad Tecnológica Nacional.

Martini, G. (2010). Estratégias de trabalho para a aprendizagem de operações com números inteiros. (Monografia para obtenção do grau de Licenciatura em Matemática). Universidade Federal do Rio Grande do Sul. Porto Alegre.

Moreira, M. A. (2003). Pesquisa em ensino: aspectos metodológicos. In M. Marco. António. Pesquisa em ensino: o Vê epistemológico de Gowin.(pp. 05-38). Porto Alegre: Actas del PIDEC. Recuperado de: httpmoreira.if.ufrgs.brpesquisaemensino.pdf

Nogueira, C. M. I., Ferreira, A. P., O. \& Oliveira, L. L. A.. (2010). Os recursos didácticos como mediadores dos processos de ensinar e aprender matemática. 
Projecto de intervenção pedagógica do programa de desenvolvimento educacional da secretaria de estado da educação do Paraná. Paraná. Recuperado de:

http://www.diaadiaeducacao.pr.gov.br/portals/pde/arquivos/2164-8.pdf

Passos, C. L. B. (2004). Recursos Didáticos na Formação de Professores de Matemática. In: VII Anais do VII Encontro Paulista de Educação Matemática: Matemática na Escola: Conteúdos e Contextos. São Paulo : SBEM/SP. pp. 01-11.

Passos, É. O., \& Takahashi, E. K. (2018). Recursos didácticos nas aulas de matemática nos anos iniciais: critérios que orientam a escolha e o uso por parte de professores. RBEP. v. 99 (n. 251), p. 172-188. Recuperado de: http://dx.doi.org/10.24109/2176-6681.rbep.99i251.3095

Pinto, J. (2003). Psicologia de aprendizagem. Concepções, Teorias e Processos. $4^{a}$ ed. Instituto de Emprego e Formacao Profissional. Recuperado de: https://comum.rcaap.pt/bitstream/10400.26/6827/1/Psicologia\%20da\%20a prendizagem\%20-\%20concep\%C3\%A7\%C3\%B5es....pdf

Prodanov, C. C. \& Freitas, E. C. (2013). Metodologia do trabalho científico: métodos e técnicas da pesquisa e do trabalho académico. Rio Grande do Sul: Feevale.

Salgado, R. d. S. (2011). O ensino de números inteiros por meio de atividades com calculadora e jogos. (Dissertação para obtenção de grau de Mestrado em Educação Matemática). Universidade do Estado do Pará, Belém. 


\section{Universidade Federal da Grande Dourados}

Silveira, P. F. (2017). Materiais manipulativos na aprendizagem de números inteiros. (Monografia para obtenção do grau de Licenciatura em Matemática). Universidade do Rio Grande do Sul.

Soares, L. H. (2007). Os Conhecimentos Prévios e o Ensino de Números Inteiros. (Dissertação para obtenção de grau de Mestrado em Ciência da Sociedade). Universidade Estadual da Paraíba, Campina Grande.

Soares, P. J. (2008). O Jogo como Recurso Didático na Apropriação dos Números Inteiros: uma experiência de sucesso. (Dissertação para obtenção de grau de Mestrado Profissional em Ensino de Matemática). Pontifícia Universidade Católica de São Paulo, São Paulo.

Tavares, J. \& Alarção, I. (2005). Psicologia do Desenvolvimento e da Aprendizagem. Coimbra: Almedina. 